APPLICATIONES MATHEMATICAE

27,3 (2000), pp. $287-308$

S. TINDEL (Villetaneuse)

\title{
SPDEs WITH PSEUDODIFFERENTIAL GENERATORS: THE EXISTENCE OF A DENSITY
}

Abstract. We consider the equation $d u(t, x)=L u(t, x)+b(u(t, x)) d t d x+$ $\sigma(u(t, x)) d W(t, x)$ where $t$ belongs to a real interval $[0, T], x$ belongs to an open (not necessarily bounded) domain $\mathcal{O}$, and $L$ is a pseudodifferential operator. We show that under sufficient smoothness and nondegeneracy conditions on $L$, the law of the solution $u(t, x)$ at a fixed point $(t, x) \in$ $[0, T] \times \mathcal{O}$ is absolutely continuous with respect to the Lebesgue measure.

1. Introduction. Stochastic Partial Differential Equations (SPDEs) of evolution type can be dealt with basically using two approaches: first, we can consider the solution $u=\{u(t, x): t \in[0, T], x \in \mathcal{O}\}$ of a SPDE, where $\mathcal{O}$ is an open domain of $\mathbb{R}^{d}$ and $T$ a positive constant, as a one-parameter process $u(t)$ with values in the Hilbert space $L^{2}(\mathcal{O})$ of square integrable functions in $\mathcal{O}$. This is the evolution system approach, widely studied by many authors (see for example Da Prato and Zabczyk [3] for a general account on this subject, and also Peszat and Zabczyk [15] for the case of the stochastic heat equation on $\mathbb{R}^{d}$ ). The other point of view is to consider $u$ as a one-dimensional multiparameter process. This is the point of view explained for example in Walsh [18], but mainly treated there in the case of the one-dimensional stochastic heat equation, that is, an evolution equation whose generator is the Laplacian $\Delta$. A way of generalizing Walsh's results to a wider class of stochastic evolution equations has been considered by Kotelenez ([8], [9]), where the infinitesimal generator is a pseudodifferential operator which generates an evolution semigroup satisfying some general smoothness conditions.

Though the semigroup approach may be a more general setting, Kotelenez's multiparameter approach allows an easier proof of various properties

2000 Mathematics Subject Classification: 60H07, 60H15.

Key words and phrases: pseudodifferential operators, stochastic partial differential equations, Malliavin's calculus. 
of the solution to the SPDE, such as space-time regularity or comparison theorems. Another advantage of the multiparameter setting is that it allows us to get quite easily some smoothness results, in the Malliavin calculus sense, for the solution $u(t, x)$ of the SPDE at a fixed point $(t, x) \in[0, T] \times \mathcal{O}$. We will use this fact to get some simple conditions on the coefficients of the equation in order to have a density for the law of $u(t, x)$ with respect to the Lebesgue measure at fixed $(t, x)$, following Pardoux and Zhang's approach [14].

Note that the existence and properties of the density for the solution to a SPDE have been addressed by many authors over the last years, after Pardoux and Zhang's paper [14], in the case of the one-dimensional stochastic heat equation: Bally and Pardoux [1] studied the $C^{\infty}$ regularity of the density, D. Márquez and M. Sanz [11] gave an expansion of the density in terms of $\varepsilon$ when the heat equation is perturbed by a small space-time white noise $\varepsilon \dot{W}$, and Donati and Pardoux [4] investigated the case of parabolic SPDEs with reflexion. Our paper also fits in a more global project of extending those density results to other kind of SPDEs, as done by Lanjri and Nualart [10] for stochastic Burgers equations, and Millet and Sanz [12] for a two-dimensional wave equation: our aim here is to get the basic existence result for the density of the law of $u(t, x)$ when $u$ is the solution to a SPDE involving a fairly general pseudodifferential operator $L$.

Notice also that the assumptions we impose on the operator $L$ in our SPDE are the same as in Kotelenez's paper [8], and will be recalled in Section 3 (hypotheses (H1) to (H6)). Moreover, the proof of the existence of a density for the law of $u(t, x)$, the solution of our SPDE at a point $(t, x) \in[0,1] \times \mathcal{O}$, will heavily rely on the positivity of $G$, the fundamental solution to the equation $\partial_{t} X=L X$. Those conditions may seem restrictive, but it is shown in [8] that they are satisfied in the following interesting cases:

1. $\mathcal{O}=\mathbb{R}, L=(-\Delta)^{\gamma / 2}$ with $\gamma \in(1,2]$.

2. $\mathcal{O} \subset \mathbb{R}^{d}$ and $L$ is the closure of a strongly elliptic operator of order $\gamma=2 m$ with $\gamma>d$.

Note also that very general conditions are given in [6] in order to ensure that a given pseudodifferential operator generates a Feller semigroup.

The paper is organized as follows: in Section 2 we recall some basic facts on Malliavin's calculus, and in Section 3 we give the general assumptions we shall make on the coefficients of the pseudodifferential equation. Section 4 is devoted to the differentiability (in the Malliavin calculus sense) of the solution $u(t, x)$ to our SPDE, and in Section 5 we prove the existence of a density for the law of $u(t, x)$. 
2. Malliavin calculus tools. We recall some basic facts on the Malliavin calculus for the space-time white noise, and give some rules that ensure the differentiability and the existence of a density for the solution of our stochastic pseudodifferential equation. In the rest of the paper, $\mathcal{O}$ denotes an open, not necessarily bounded, set in $\mathbb{R}^{d}$, satisfying the cone condition, that is,

$$
\inf _{\varrho>0} \inf _{x \in \mathcal{O}} \frac{\lambda(\mathcal{O} \cap B(x, \varrho))}{\varrho^{d}}>0,
$$

where

$$
B(x, \varrho)=\left\{y \in \mathbb{R}^{d}:|x-y|<\varrho\right\} .
$$

For simplicity of notation, we assume that our probability space $(\Omega, \mathcal{F}, P)$ is as follows: $\Omega=C([0, T] \times \mathcal{O})$, the set of continuous functions on $[0, T]$ $\times \mathcal{O}$, and $\mathcal{F}$ is the Borel field of $\Omega$. The probability measure $P$ is such that the family $\{W(A): A \in \mathcal{B}([0, T] \times \mathcal{O}), \lambda(A)<\infty\}$ is a centered Gaussian family whose covariance function is given by

$$
E[W(A) W(B)]=\lambda(A \cap B),
$$

where $\lambda$ denotes the Lebesgue measure on $[0, T] \times \mathcal{O}$, and $\mathcal{B}([0, T] \times \mathcal{O})$ is the Borel field on $[0, T] \times \mathcal{O}$. We also consider the filtration $\left\{\mathcal{F}_{t}: t \geq 0\right\}$ defined by

$$
\mathcal{F}_{t}=\sigma\{W(A): A \in \mathcal{B}([0, t] \times \mathcal{O}), \lambda(A)<\infty\} \vee \mathcal{N},
$$

where $\mathcal{N}$ is the class of $P$-null sets of $\mathcal{F}$.

Set $H=L^{2}([0, T] \times \mathcal{O})$. For any $h \in H$, we call $W(h)$ the Wiener integral of $H$ on $[0, T] \times \mathcal{O}$, that is (the material on the Malliavin calculus of this section is taken mainly from [13], and we refer in particular to this book for a more complete account on Wiener integrals),

$$
W(h)=\int_{0}^{T} \int_{\mathcal{O}} h(t, x) d W(t, x) .
$$

The space $\mathcal{S}$ of smooth functionals on $\Omega$ is the set of random variables of the form

$$
F=l\left(W\left(h_{1}\right), \ldots, W\left(h_{n}\right)\right),
$$

where $n \in \mathbb{N}, h_{i} \in H, l \in C_{\mathrm{b}}^{\infty}\left(\mathbb{R}^{n}\right)$. For such a variable, we can define the derivative $D F$ as an $H$-valued random variable by

$$
D_{t, x} F=\sum_{i=1}^{n} \frac{\partial l}{\partial x_{i}}\left(W\left(h_{1}\right), \ldots, W\left(h_{n}\right)\right) h_{i}(t, x), \quad(t, x) \in[0, T] \times \mathcal{O} .
$$

For $p>1$, we denote by $\mathbb{D}^{1, p}$ the closure of $\mathcal{S}$ with respect to the seminorm

$$
\|F\|_{1, p}=\left\{E\left[|F|^{p}\right]+E\left[\|D F\|_{H}^{p}\right]\right\}^{1 / p} .
$$


If $G$ is a $V$-valued random variable, where $V$ is a separable Hilbert space, we can also define the derivative of $G$, and the corresponding sets $\mathbb{D}^{1, p}(V)$ for $p>1$. The following chain rule for a Lipschitz function can be shown (see e.g. [13, Prop. 1.2.3]):

TheOREM 2.1. Let $\varphi: \mathbb{R} \rightarrow \mathbb{R}$ be a Lipschitz function with Lipschitz constant $c$, and $F$ a random variable that belongs to $\mathbb{D}^{1,2}$. Then $\varphi(F) \in \mathbb{D}^{1,2}$ and there exists a random variable $G$ bounded by $c$ such that

$$
D(\varphi(F))=G D F .
$$

Let $\delta$ be the adjoint of the derivative operator as an unbounded operator on $L^{2}(\Omega)$. The domain of $\delta$ is denoted by $\operatorname{Dom} \delta$. The space $L_{\mathrm{a}}^{2}$ of $\mathcal{F}_{t}$-adapted, measurable and square integrable random fields is included in $\operatorname{Dom} \delta$, and $\delta$ restricted to $L_{\mathrm{a}}^{2}$ coincides with the Itô integral with respect to $W$. Notice that $\mathbb{D}^{1,2}(H) \subset \operatorname{Dom} \delta$. Moreover, if $u \in \mathbb{D}^{1,2}(H)$, then $\delta(u) \in \mathbb{D}^{1,2}$ and the following commutation relation between $D$ and $\delta$ holds:

$$
D_{t, x}(\delta(u))=u(t, x)+\int_{0}^{t} \int_{\mathcal{O}} D_{t, x} u(s, y) d W(s, y) .
$$

We will use the following two rules to get our density result (see $\mathrm{Nu}-$ alart [13] for the proofs).

Theorem 2.2. Let $\left\{F_{n}: n \geq 1\right\}$ be a family of elements of $\mathbb{D}^{1,2}$ converging to $F$ in $L^{p}(\Omega)$ for some $p>1$. Suppose that $\left\{D F_{n}: n \geq 1\right\}$ is a bounded family in $L^{p}(\Omega ; H)$. Then $F \in \mathbb{D}^{1, p}, F_{n} \in \mathbb{D}^{1, p}$ for every $n \geq 1$, and there exists a subsequence of $\left\{D F_{n}: n \geq 1\right\}$ converging to $D F$ in the weak topology of $L^{p}(\Omega ; H)$.

Theorem 2.3. Let $F$ be a real random variable defined on $\Omega$. Suppose that $F \in \mathbb{D}^{1,2}$ and that $\|D F\|_{H}>0$ almost surely. Then the measure $P \circ F^{-1}$ is absolutely continuous with respect to the Lebesgue measure.

3. Pseudodifferential equations. We state here some results proved by Kotelenez [9], also cited by Kallianpur and Xiong [7], on the solution of a stochastic pseudodifferential equation, giving sometimes a slightly different proof of the result. Let $C_{\mathrm{c}}^{\infty}(\mathcal{O})$ be the set of smooth functions with compact support in $\mathcal{O}$. Throughout the paper, $c$ will be a constant that can change from line to line. We will sometimes stress the dependence of $c$ on the parameters of the problem. Let us recall the definition of a pseudodifferential operator, given for example in Treves [17].

Definition. Let $\gamma \in \mathbb{R}$. A pseudodifferential operator of order $\gamma$ is an operator defined on $C_{\mathrm{c}}^{\infty}(\mathcal{O})$ by 


$$
L f(x)=(2 \pi)^{-d} \int_{\mathcal{O} \mathbb{R}^{d}} e^{i(x-y) v} a(x, y, v) f(y) d y d v, \quad f \in C_{\mathrm{c}}^{\infty}(\mathcal{O}),
$$

where $a: \mathcal{O} \times \mathcal{O} \times \mathbb{R}^{d} \rightarrow \mathbb{C}$ is a smooth function with compact support, and for any compact set $K \subset \mathcal{O} \times \mathcal{O}$ and multiindices $\beta_{1}, \beta_{2}, \beta_{3}$, there exists a constant $c=c\left(C, \beta_{1}, \beta_{2}, \beta_{3}\right)$ such that

$$
\left|\partial_{x}^{\beta_{1}} \partial_{y}^{\beta_{2}} \partial_{v}^{\beta_{3}} a(x, y, v)\right| \leq c(1+|v|)^{\gamma-\left|\beta_{3}\right|}
$$

for any $(x, y) \in K, v \in \mathbb{R}^{d}$.

Let $L$ be a pseudodifferential operator, $b, \sigma: \mathbb{R} \rightarrow \mathbb{R}$ two functions, and $d W(t, x)$ the space-time white noise on $[0, T] \times \mathbb{R}^{d}$. We consider the following $\operatorname{SPDE}$ for $(t, x) \in[0, T] \times \mathcal{O}$ :

$$
\left\{\begin{array}{l}
d u(t, x)=[L u(t, x)+b(u(t, x))] d t+\sigma(u(t, x)) d W(t, x) \\
u(0, x)=u_{0}(x)
\end{array}\right.
$$

where $u_{0}$ is a continuous function from $\mathcal{O}$ to $\mathbb{R}$. We will make the following assumptions on $L$ and the coefficients of (2):

(H1) $L$ is a pseudodifferential operator of order $\gamma>d$.

(H2) $L$ generates a semigroup $\{S(t): t \geq 0\}$ of linear operators from $C_{\mathrm{c}}^{\infty}(\mathcal{O})$ to $C(\mathcal{O})$ which has a kernel function $G(t, x, \xi)$, that is, $(S(t) f)(x)=\int_{\mathcal{O}} G(t, x, \xi) f(\xi) d \xi$.

The solution to (2) has to be interpreted in the mild sense: we say that $u$ is a solution to the stochastic pseudodifferential equation if it is an $\mathcal{F}_{t}$-adapted process on $[0, T] \times \mathcal{O}$ satisfying

$$
\begin{aligned}
u(t, x)= & \int_{\mathcal{O}} G(t, x, \xi) u_{0}(\xi) d \xi+\int_{0}^{t} \int_{\mathcal{O}} G(t-\theta, x, \xi) b(u(\theta, \xi)) d \theta d \xi \\
& +\int_{0}^{t} \int_{\mathcal{O}} G(t-\theta, x, \xi) \sigma(u(\theta, \xi)) d W(\theta, \xi) .
\end{aligned}
$$

Note that in Kotelenez's general case, the pseudodifferential generator $L$ is allowed to be time-dependent. However, we will have to apply a comparison result for solutions of (2), which was obtained in [9] only for a fixed $L$.

The following regularity conditions on $G$ and its Fourier transform are needed in order to get the existence, uniqueness and continuity of the solution.

(H3) For any $T>0$, there exists a constant $c \equiv c(T)<\infty$ such that for any $0<t \leq T$,

$$
\int_{\mathcal{O}}|G(t, x, \xi)| d \xi \leq c
$$


(H4) There is a symbol $g(t, \xi, \bar{\xi})$ for $0<t \leq T, \xi \in \mathcal{O}, \bar{\xi} \in \mathbb{R}^{d}$, such that, for a constant $c \equiv c(T)$,

$$
G(t, x, \xi)=\frac{1}{(2 \pi)^{d}} \int_{\mathbb{R}^{d}} \exp (i(x-\xi) \bar{\xi}) g(t, x, \bar{\xi}) d \bar{\xi}
$$

and

$$
\int_{\mathbb{R}^{d}}|\bar{\xi}|^{\alpha}\left|g\left(t, \xi, t^{-1 / \gamma} \bar{\xi}\right)\right| d \bar{\xi} \leq c
$$

for all $0 \leq \alpha \leq \gamma-d, 0<t \leq T, \xi \in \mathcal{O}$.

There is a constant $c \equiv c(\gamma)$ such that

$$
\left|\int_{\mathbb{R}^{d}} \exp (i(x-\xi) \bar{\xi})[g(s+s r, \xi, \bar{\xi})-g(s r, \xi, \bar{\xi})] d \bar{\xi}\right| \leq c s^{-d / \gamma} r^{-1}
$$

for all $0<s<s+s r<T, x, \xi \in \mathcal{O}$.

(H6) For all $T>0$ there exist two integrable functions $p$ and $q$ defined on $\mathbb{R}^{d}$ such that for $0<t \leq T$ and $x, \xi \in \mathcal{O}$, and any compact $K \subset \mathbb{R}^{d}$,

$$
\begin{gathered}
q\left((x-\xi) t^{-1 / \gamma}\right) \leq t^{d / \gamma} G(t, x, \xi) \leq p\left((x-\xi) t^{-1 / \gamma}\right), \\
\sup _{x \in \mathbb{R}^{d}} p(x) \leq c<\infty, \quad \inf _{x \in K} q(x)>0 .
\end{gathered}
$$

The use of the positive function $q$ is an additional hypothesis with respect to the original ones. We need that positivity condition on $G$ in order to get our density result. Finally, we will need the usual Lipschitz conditions on the coefficients of the equation, and a boundedness condition on $u_{0}$ :

There exists a constant $c$ such that

$$
|b(x)-b(y)|+|\sigma(x)-\sigma(y)| \leq c|x-y|, \quad \sup _{x \in \mathcal{O}} u_{0}(x)<\infty,
$$

and $u_{0} \in C(\mathcal{O})$.

Under these hypotheses, the following existence and uniqueness theorem is shown in [9]. In order to fix our notations for the remainder of the paper, we will give here a slightly different proof of the first part of the result. Note that the regularity conditions on the Fourier transform are only needed to prove the continuity result for the solution, and that the norms $|\cdot| \mathbb{F}_{\lambda}$ used in our proof are similar to the one used e.g. in Seidler [16, Proposition 4.1].

TheOREM 3.1. Suppose that the assumptions (H1), (H2), (H3), (H6) and (H7) are satisfied. Then:

(i) There exists a unique $\mathcal{F}_{t}$-adapted mild solution to (2), in the sense given by (3).

(ii) The solution $u$ satisfies, for any $p>1$,

$$
\sup _{t \in[0, T]} \sup _{x \in \mathcal{O}} E\left[|u(t, x)|^{p}\right]<\infty .
$$


(iii) Under the aditional hypotheses (H4) and (H5), the solution $u$ satisfies, for any $n$ large enough so that $\widehat{n}=[2(n-1) /(n-2)]-1$ is such that $0<\widehat{n}<\gamma / d$

$$
\sup _{t, t+s \in[0, T]} \sup _{x, x+h \in \mathcal{O}} E\left[|u(t+s, x+h)-u(t, x)|^{n}\right] \leq c s^{\left(1-\frac{d}{\gamma} \widehat{n}\right) \frac{n-2}{2}}+|h|^{\frac{d}{\gamma} \frac{n-2}{2}},
$$

where $c \equiv c(T, p)$ is a positive constant.

(iv) Under hypotheses (H1)-(H7), the solution is almost surely Hölder continuous in $(t, x) \in[0, T] \times \mathcal{O}$ with exponent

$$
\left(\left[\frac{1}{2}\left(1-\frac{d}{\gamma}\right)\right] \wedge\left[\frac{\gamma-d}{2}\right] \wedge 1\right)-\varepsilon
$$

for any $\varepsilon>0$.

Pro of of (i). Let $\mathbb{E}$ be the set of $\mathcal{F}_{t}$-adapted measurable processes $\Xi=\{\Xi(t, x): 0<t \leq T, x \in \mathcal{O}\}$ such that $\Xi(0, x)=v_{0}(x)$ for a continuous bounded initial condition $v_{0}$, and

$$
\sup _{t \in[0, T]} \sup _{x \in \mathcal{O}} E\left[|\Xi(t, x)|^{2}\right]<\infty .
$$

We set, for $v \in \mathbb{E}$,

$$
\mathcal{A} v=v_{0}+G(v)+H(v),
$$

where

$$
\begin{aligned}
G(v)(t, x) & =\int_{0}^{t} \int_{\mathcal{O}} G(t-\theta, x, \xi) b(v(\theta, \xi)) d \theta d \xi, \\
H(v)(t, x) & =\int_{0}^{t} \int_{\mathcal{O}} G(t-\theta, x, \xi) \sigma(v(\theta, \xi)) d W(\theta, \xi) .
\end{aligned}
$$

STEP 1. Let us show that $\mathcal{A}$ is a mapping from $\mathbb{E}$ to $\mathbb{E}$. For a constant $c$,

$$
\begin{aligned}
\sup _{t \in[0, T]} \sup _{x \in \mathcal{O}} E\left[|\mathcal{A} v(t, x)|^{2}\right] \leq & c\left(\sup _{x \in \mathcal{O}}\left|v_{0}(x)\right|+\sup _{t \in[0, T]} \sup _{x \in \mathcal{O}} E\left[|G(v)(t, x)|^{2}\right]\right. \\
& \left.+\sup _{t \in[0, T]} \sup _{x \in \mathcal{O}} E\left[|H(v)(t, x)|^{2}\right]\right) .
\end{aligned}
$$

The first term on the right hand side is bounded, by our assumption on $v_{0}$. Moreover, applying Schwarz's inequality, we obtain, for every $\varepsilon \in(0,1)$,

$$
\begin{aligned}
E\left[|G(v)(t, x)|^{2}\right] \leq & \left(\int_{0}^{t} \int_{\mathcal{O}} G^{2 \varepsilon}(t-\theta, x, \xi) d \xi d \theta\right) \\
& \times\left(\iint_{0}^{t} G^{2(1-\varepsilon)}(t-\theta, x, \xi) E\left[\mid b\left(\left.v(\theta, \xi)\right|^{2}\right] d \xi d \theta\right) .\right.
\end{aligned}
$$


Choose $\varepsilon=1 / 2$. Then, by (H3) and (H7), we get

$$
E\left[|G(v)(t, x)|^{2}\right] \leq c\left(1+\sup _{\theta \in[0, T]} \sup _{x \in \mathcal{O}} E\left[|v(\theta, \xi)|^{2}\right]\right) .
$$

Thus, the process $\{G(v)(t, x): 0<t \leq T, x \in \mathcal{O}\}$ satisfies inequality (4) whenever $v \in \mathbb{E}$. Let us get a bound for the stochastic integral part. From the isometry property for a martingale measure (see Walsh [18]) and Schwarz's inequality, we get, using hypothesis (H7),

$$
\begin{aligned}
E\left[|H(v)(t, x)|^{2}\right] & =\int_{0}^{t} \int_{\mathcal{O}} G^{2}(t-\theta, x, \xi) E\left[\sigma^{2}(v(\theta, \xi))\right] d \theta d \xi \\
& \leq c\left(1+\sup _{t \in[0, T]} \sup _{x \in \mathcal{O}} E\left[|v(\theta, \xi)|^{2}\right]\right)\left(\int_{0}^{t} \int_{\mathcal{O}} G^{2}(t-\theta, x, \xi) d \theta d \xi\right) .
\end{aligned}
$$

Note that, from (H3) and (H6), we have (recall that $c$ is a constant that can change from line to line)

$$
\begin{aligned}
\int_{0}^{t} \int_{\mathcal{O}} G^{2}(t- & \theta, x, \xi) d \theta d \xi \\
& =\int_{0}^{t}(t-\theta)^{-d / \gamma} \int_{\mathcal{O}}(t-\theta)^{d / \gamma} G(t-\theta, x, \xi) G(t-\theta, x, \xi) d \theta d \xi \\
& \leq \int_{0}^{t}(t-\theta)^{-d / \gamma}\left(\int_{\mathcal{O}} p\left((t-\theta)^{-d / \gamma}(x-\xi)\right) G(t-\theta, x, \xi) d \xi\right) d \theta \\
& \leq c \int_{0}^{t}(t-\theta)^{-d / \gamma}\left(\int_{\mathcal{O}} G(t-\theta, x, \xi) d \xi\right) d \theta \\
& \leq c \int_{0}^{t}(t-\theta)^{-d / \gamma} d \theta \leq c,
\end{aligned}
$$

since $d<\gamma$. Hence,

$$
\sup _{t \in[0, T]} \sup _{x \in \mathcal{O}} E\left[|H(v)(t, x)|^{2}\right] \leq c\left(1+\sup _{t \in[0, T]} \sup _{x \in \mathcal{O}} E\left[|v(t, x)|^{2}\right]\right) .
$$

We have thus proved that $\mathcal{A}$ maps $\mathbb{E}$ in $\mathbb{E}$.

STEP 2. Consider now a parameter $\lambda>0$ and the set $\mathbb{F}_{\lambda}$ of $\mathcal{F}_{t}$-adapted measurable processes $v=\{v(t, x): t \in[0, T], x \in \mathcal{O}\}$ equipped with the norm

$$
|v|_{\mathbb{F}_{\lambda}}^{2}=\int_{0}^{T} e^{-\lambda t} \sup _{x \in \mathcal{O}} E\left[|v(t, x)|^{2}\right] d t .
$$


Notice that $v_{0} \in \mathbb{F}_{\lambda}$ for every $\lambda>0$ whenever $v_{0}$ is a bounded continuous function defined on $\mathcal{O}$, and therefore, from Step 1, the sequence $\left\{\mathcal{A}^{k} v_{0}\right.$ : $k \geq 0\}$ is in $\mathbb{F}_{\lambda}$ for any $\lambda>0$. Hence, if we show that for sufficiently large $\lambda$, $\mathcal{A}$ is a contraction in $\mathbb{F}_{\lambda}$, we will get the existence and uniqueness of the solution to (2) by a fixed point argument. Let $u$ and $v$ be processes in $\mathbb{F}_{\lambda}$. We have

$$
\mathcal{A} v-\mathcal{A} u=G(v)-G(u)+H(v)-H(u) .
$$

For the deterministic part of the right hand side of (5), we get, as in Step 1, using hypotheses (H3), (H6) and (H7),

$$
\begin{aligned}
E\left[|(G(v)-G(u))(t, x)|^{2}\right] & \\
\leq & \left(\int_{0}^{t} \int_{\mathcal{O}} G(t-\theta, x, \xi) d \xi d \theta\right) \\
& \quad \times\left(\int_{0}^{t} \int_{\mathcal{O}} G(t-\theta, x, \xi) E\left[|b(v(\theta, \xi))-b(u(\theta, \xi))|^{2}\right] d \xi d \theta\right) \\
\leq & c \int_{0}^{t} \sup _{x \in \mathcal{O}} E\left[|v(\theta, x)-u(\theta, x)|^{2}\right] d \theta .
\end{aligned}
$$

For the stochastic part, we also get, as in Step 1,

$$
\begin{aligned}
& E\left[|(H(v)-H(u))(t, x)|^{2}\right] \\
& \quad \leq c \int_{0}^{t} \sup _{x \in \mathcal{O}} E\left[|\sigma(v(\theta, \xi))-\sigma(u(\theta, \xi))|^{2}\right]\left(\int_{\mathcal{O}} G^{2}(t-\theta, x, \xi) d \xi\right) d \theta \\
& \quad \leq c \int_{0}^{t}(t-\theta)^{-d / \gamma} \sup _{x \in \mathcal{O}} E\left[|v(\theta, \xi)-u(\theta, \xi)|^{2}\right] d \theta .
\end{aligned}
$$

Hence

$$
\begin{aligned}
|\mathcal{A} v-\mathcal{A} u|_{\mathbb{F}_{\lambda}}^{2} & =\int_{0}^{T} e^{-\lambda t} \sup _{x \in \mathcal{O}} E\left[|\mathcal{A} v(t, x)-\mathcal{A} u(t, x)|^{2}\right] d t \\
& \leq c \int_{0}^{T} e^{-\lambda t}\left(\int_{0}^{t}(t-\theta)^{-d / \gamma} \sup _{x \in \mathcal{O}} E\left[|v(\theta, x)-u(\theta, x)|^{2}\right] d \theta\right) d t .
\end{aligned}
$$

Set, for $0 \leq \theta \leq T$,

$$
\varphi(\theta)=\sup _{x \in \mathcal{O}} E\left[|v(\theta, x)-u(\theta, x)|^{2}\right],
$$


and note that $|v-u|_{\mathbb{F}_{\lambda}}=\int_{0}^{T} e^{-\lambda \theta} \varphi(\theta) d \theta$. We have

$$
\begin{aligned}
|\mathcal{A} v-\mathcal{A} u|_{\mathbb{F}_{\lambda}}^{2} & \leq c \int_{0}^{T} e^{-\lambda t}\left(\int_{0}^{t} s^{-d / \gamma} \varphi(t-s) d s\right) d t \\
& =c \int_{0}^{T} s^{-d / \gamma}\left(\int_{s}^{T} e^{-\lambda t} \varphi(t-s) d t\right) d s \\
& \leq c\left(\int_{0}^{\infty} e^{-\lambda s} s^{-d / \gamma} d s\right)|v-u|_{\mathbb{F}_{\lambda}}^{2},
\end{aligned}
$$

and hence, for sufficiently large $\lambda, \mathcal{A}$ is a contraction on $\mathbb{F}_{\lambda}$, which ends the proof.

REMARK 3.2. It is shown in [8] that for any $(t, x) \in[0, T] \times \mathcal{O}$ and $p>1$, $L^{p}-\lim _{n \rightarrow \infty} u^{n}(t, x)=u(t, x)$, where $u^{n}=\mathcal{A}^{n} u_{0}$ and $L^{p}$-lim stands for the limit in $L^{p}(\Omega)$.

Note that under hypothesis (H6), by positivity of the kernel $G$, the semigroup $\{S(t): t \geq 0\}$ is positivity preserving, and hence we can apply a slight variation of Theorem 3.5 in [9] to compare solutions of equation (2).

Theorem 3.3. Suppose (H1)-(H6). For $i=1,2$, let $B_{i}: \Omega \times[0, T] \times$ $\mathcal{O} \rightarrow \mathbb{R}$ be an $\mathcal{F}_{t}$-adapted bounded process. Let $u_{0}^{i}$ and $\sigma$ be resp. two initial conditions and a diffusion coefficient satisfying $(\mathrm{H} 7)$. Let $u_{i}, i=1,2$, be the solutions to the equations

$$
\left\{\begin{array}{l}
d u(t, x)=\left[L u(t, x)+B_{i}(t, x) u(t, x)\right] d t+\sigma(u(t, x)) d W(t, x), \\
u(0, x)=u_{0}^{i}(x) .
\end{array}\right.
$$

Suppose further that

$$
B_{1} \leq B_{2}, \quad u_{0}^{1} \leq u_{0}^{2} .
$$

Then almost surely, for any $(t, x) \in[0, T] \times \mathcal{O}$, we have

$$
u_{1}(t, x) \leq u_{2}(t, x) .
$$

Lastly, we recall the Garsia-type lemma given by Kallianpur and Xiong [7] for a general bounded domain in $\mathbb{R}^{d}$ satisfying the cone condition. We give here a version of this theorem suited for stochastic processes.

THEOREM 3.4. Let $Q$ be an open bounded domain of $\mathbb{R}^{d}$ satisfying the cone condition. Let $x=\{x(y): y \in Q\}$ be a measurable process such that for a constant $p>1$ and for any $y_{1}, y_{2} \in Q$,

$$
E\left[\left|x\left(y_{1}\right)-x\left(y_{2}\right)\right|^{p}\right] \leq c\left|y_{1}-y_{2}\right|^{q},
$$

where $q>d$. Then for any $0<m<q-d$, there exist some positive constants $c_{1}, c_{2}$ and a random variable $\Gamma$ such that

$$
\left|x\left(y_{1}\right)-x\left(y_{2}\right)\right|^{p} \leq c_{1}\left|y_{1}-y_{2}\right|^{m} \Gamma, \quad E[\Gamma] \leq c_{2} .
$$




\section{Malliavin calculus for a pseudodifferential equation}

4.1. Differentiability of the solution. We first establish, using Theorem 2.2, the differentiability (in the Malliavin calculus sense) of the solution to equation (2).

Proposition 4.1. Under hypotheses (H1)-(H7), the solution $u(t, x)$ of (2) is an element of $\mathbb{D}^{1,2}$ for any $(t, x) \in[0, T] \times \mathcal{O}$, and the derivative satisfies, for $(s, y) \in[0, t] \times \mathcal{O}$,

$$
\begin{aligned}
D_{s, y} u(t, x)= & \int_{s}^{t} \int_{\mathcal{O}} G(t-\theta, x, \xi) B(\theta, \xi) D_{s, y} u(\theta, \xi) d \theta d \xi \\
& +\int_{s}^{t} \int_{\mathcal{O}} G(t-\theta, x, \xi) S(\theta, \xi) D_{s, y} u(\theta, \xi) d W(\theta, \xi) \\
& +\mathbf{1}_{[0, t]}(s) G(t-s, x, y) \sigma(u(s, y)),
\end{aligned}
$$

where $B$ and $S$ are bounded $\mathcal{F}_{t}$-adapted processes. Moreover, $D_{s, y} u(t, x)=0$ for $s>t$.

Proof. Let $u_{0}$ be an initial condition satisfying (H7), and set $u_{n}=$ $\mathcal{A}^{n} u_{0}$. According to Remark 3.2, $L^{p}-\lim _{n \rightarrow \infty} u_{n}(t, x)=u(t, x)$ for every $(t, x) \in[0, T] \times \mathcal{O}$. Let $\mathbb{M}^{1,2}$ be the set of processes $u(t, x)$ such that $u(t, x) \in$ $\mathbb{D}^{1,2}$ for any $(t, x) \in[0, T] \times \mathcal{O}$ and

$$
\|u\|_{\mathbb{M}^{1,2}}^{2} \equiv \sup \left\{E\left[|u(t, x)|^{2}\right]+E\left[|D u(t, x)|_{H}^{2}\right]: t \in[0, T], x \in \mathcal{O}\right\}<\infty .
$$

To prove the differentiability of the solution, applying Theorem 2.2, it is sufficient to prove that $u_{n} \in \mathbb{M}^{1,2}$ for any $n \geq 0$, and that for any $(t, x) \in$ $[0, T] \times \mathcal{O},\left\{\left\|u_{n}(t, x)\right\|_{1,2}: n \geq 0\right\}$ is bounded. Let us show it inductively: if $u_{n} \in \mathbb{D}^{1,2}$, then

$$
\begin{aligned}
u_{n+1}(t, x)= & \mathcal{A} u_{n}(t, x) \\
= & \int_{\mathcal{O}} G(t, x, \xi) u_{0}(\xi) d \xi+\int_{0}^{t} \int_{\mathcal{O}} G(t-\theta, x, \xi) b\left(u_{n}(\theta, \xi)\right) d \theta d \xi \\
& +\int_{0}^{t} \int_{\mathcal{O}} G(t-\theta, x, \xi) \sigma\left(u_{n}(\theta, \xi)\right) d W(\theta, \xi) .
\end{aligned}
$$

If $u_{n} \in \mathbb{M}^{1,2}$, we can differentiate equation (6), and using the commutation relation (1) and the composition relation of Theorem 2.1 we see that

$$
D u_{n+1}=\left\{D_{s, y} u_{n+1}(t, x): t \in[0, T], y, x \in \mathcal{O}, 0 \leq s \leq t\right\}
$$

satisfies 


$$
\begin{aligned}
D_{s, y} u_{n+1}(t, x)= & \int_{s}^{t} \int_{\mathcal{O}} G(t-\theta, x, \xi) B_{n}(\theta, \xi) D_{s, y} u_{n}(\theta, \xi) d \theta d \xi \\
& +\int_{s}^{t} \int_{\mathcal{O}} G(t-\theta, x, \xi) S_{n}(\theta, \xi) D_{s, y} u_{n}(\theta, \xi) d W(\theta, \xi) \\
& +\mathbf{1}_{[0, t]}(s) G(t-s, x, y) \sigma\left(u_{n}(s, y)\right),
\end{aligned}
$$

where $B_{n}$ and $S_{n}$ are two $\mathcal{F}_{t}$-adapted processes bounded by the Lipschitz constant of $b$ and $\sigma$. Recall also that if $x_{t}$ is an $\mathcal{F}_{t}$-adapted process such that $x_{t} \in \mathbb{D}^{1,2}$, then $D_{s} x_{t}=0$ if $s>t$, and thus the integrals involved in the definition of $D_{s, y} u_{n+1}(t, x)$ can be taken from $s$ to $t$ instead of from 0 to $t$.

Using hypotheses (H6) and (H3), we have (recall that $c$ is a constant that can change from line to line)

$$
\begin{aligned}
& E\left[\int_{0}^{t} \int_{\mathcal{O}} G^{2}(t-s, x, y) \sigma^{2}\left(u_{n}(s, y)\right) d s d y\right] \\
& \quad \leq c E\left[\int_{0}^{t} \int_{\mathcal{O}} G^{2}(t-s, x, y)\left(1+u_{n}^{2}(s, y)\right) d s d y\right] \\
& \quad \leq c\left(1+\int_{0}^{t}(t-s)^{-d / \gamma} \sup _{y \in \mathcal{O}} E\left[\left|u_{n}^{2}(s, y)\right|\right]\left(\int_{\mathcal{O}} G(t-s, x, y) d y\right) d s\right) \\
& \quad \leq c\left(1+\int_{0}^{t}(t-s)^{-d / \gamma} V_{n}(s) d s\right),
\end{aligned}
$$

where we have set

$$
V_{n}(s)=\sup _{y \in \mathcal{O}}\left\|u_{n}(s, y)\right\|_{1,2}^{2}
$$

We also have, using the same arguments as for inequality (7),

$$
\begin{aligned}
& E\left[\int_{0}^{t} \int_{\mathcal{O}}\left(\int_{0}^{t} \int_{\mathcal{O}} G(t-\theta, x, \xi) S_{n}(\theta, \xi) D_{s, y} u_{n}(\theta, \xi) d W(\theta, \xi)\right)^{2} d s d y\right] \\
& \quad=\int_{0}^{t} \int_{\mathcal{O}}\left(\int_{0}^{t} \int_{\mathcal{O}} G^{2}(t-\theta, x, \xi) E\left[\left|S_{n}(\theta, \xi) D_{s, y} u_{n}(\theta, \xi)\right|^{2}\right] d \theta d \xi\right) d s d y \\
& \quad \leq c \int_{0}^{t} \int_{\mathcal{O}}^{t}\left(\int_{0}^{t} \int_{\mathcal{O}} G^{2}(t-\theta, x, \xi) E\left[\left|D_{s, y} u_{n}(\theta, \xi)\right|^{2}\right] d \theta d \xi\right) d s d y \\
& \leq c \int_{0}^{t} \int_{\mathcal{O}} G^{2}(t-\theta, x, \xi)\left|D u_{n}(\theta, \xi)\right|_{H}^{2} d \theta d \xi \leq c \int_{0}^{t}(t-s)^{-d / \gamma} V_{n}(s) d s .
\end{aligned}
$$


Similarly, we can prove, as in Theorem 3.1, that

$$
\begin{aligned}
E\left[\int_{0}^{t} \int_{\mathcal{O}}\left(\int_{0}^{t} \int_{\mathcal{O}} G(t-\theta, x, \xi) B_{n}(\theta, \xi) D_{s, y} u_{n}(\theta, \xi) d \theta d \xi\right)^{2} d s d y\right] \\
\leq c \int_{0}^{t} \int_{\mathcal{O}} V_{n}(\theta) d \theta .
\end{aligned}
$$

Altogether, from (7)-(9) we deduce that for a constant $c$,

$$
V_{n+1}(t) \leq c\left(1+\int_{0}^{t} V_{n}(\theta)(t-\theta)^{-d / \gamma} d \theta\right),
$$

and using Kotelenez's result [8, Lemma 1.8], we conclude that there exist some constants $c>0, k \geq 1$ such that for any $n \geq 1, t \in[0, T]$,

$$
V_{n+m k}(t) \leq c^{m}\left(1+\int_{0}^{t} V_{n}(\theta) \frac{(t-\theta)^{m-1}}{(m-1) !} d \theta\right),
$$

which gives directly

$$
\sup _{n \geq 0}\left\|u_{n}\right\|_{\mathbb{M}^{1,2}} \leq c .
$$

Theorem 2.2 now shows that $u \in \mathbb{M}^{1,2}$, and applying the derivation operator to (3), we get the equation satisfied by $D u$.

Let us show a continuity property of $D u(t, x)$. Notice that this proof is not given in [14]. Moreover, the results on regularity of the solutions with respect to a parameter for Banach valued solutions of SPDEs given in [2] cannot be directly applied here, since we are not dealing with an evolution equation with initial condition depending on a parameter, and it seems more convenient to show directly the continuity of the process $r_{s}$ defined below.

Lemma 4.2. Assume hypotheses $(\mathrm{H} 1)-(\mathrm{H} 7)$, and $f i x(t, x) \in(0, T] \times \mathcal{O}$. For an open set $Q \subset \mathcal{O}$, set

$$
r_{s}(t, x)=\int_{Q} D_{s, y} u(t, x) d y
$$

for $s \leq t$. Then the process $r_{s}(t, x)$ is almost surely continuous in $s$ for $s \in[0, t / 2]$.

Pro of. We use the Kolmogorov criterion.

STEP 1. We know that $r_{s}(t, x)$ satisfies, for $s \leq t$,

$$
r_{s}(t, x)=\int_{Q} G(t-s, x, y) \sigma(u(s, y)) d y
$$




$$
\begin{aligned}
& +\int_{s}^{t} \int_{\mathcal{O}} G(t-\theta, x, \xi) B(\theta, \xi) r_{s}(\theta, \xi) d \theta d \xi \\
& +\int_{s}^{t} \int_{\mathcal{O}} G(t-\theta, x, \xi) S(\theta, \xi) r_{s}(\theta, \xi) d W(\theta, \xi) .
\end{aligned}
$$

Define $X=\left\{X_{s}(l, z): 0 \leq s \leq t / 2,0 \leq l \leq t, z \in \mathcal{O}\right\}$ by $X_{s}(l, z)=0$ if $l \leq s$, and for any $l, z \in[s, t] \times \mathcal{O}$, by the equation

$$
\begin{aligned}
X_{s}(l, z)= & \left.\int_{Q} G(t-s, x, y) \sigma(s, y)\right) d y \\
& +\int_{s}^{l} \int_{\mathcal{O}} G(l-\lambda, z, \zeta) B(\lambda, \zeta) X_{s}(\lambda, \zeta) d \lambda d \zeta \\
& +\int_{s}^{l} \int_{\mathcal{O}} G(l-\lambda, z, \zeta) S(\lambda, \zeta) X_{s}(\lambda, \zeta) d W(\lambda, \zeta) .
\end{aligned}
$$

Then $r_{s}(t, x)=X_{s}(t, x)$. We will estimate the moments of $X_{s}(t, x)$. Let $s_{1}, s_{2} \in[0, t / 2]$, and suppose $s_{1}>s_{2}$. It can be shown easily that for every $n>1$,

$$
\sup _{s \in[0, t / 2]} \sup _{l \in[s, t]} \sup _{z \in \mathcal{O}} E\left[\left|X_{s}(l, z)\right|^{n}\right] \leq c_{n} .
$$

Then, for any $n>1$,

$$
E\left[\left|X_{s_{2}}(l, z)-X_{s_{1}}(l, z)\right|^{n}\right] \leq \sum_{i=1}^{6} I_{i}(n)
$$

with

$$
\begin{aligned}
& I_{1}(n)=E\left[\left|\int_{Q} G\left(t-s_{2}, x, y\right)\left(\sigma\left(u\left(s_{2}, y\right)\right)-\sigma\left(u\left(s_{1}, y\right)\right)\right) d y\right|^{n}\right], \\
& I_{2}(n)=E\left[\left|\int_{Q}\left(G\left(t-s_{2}, x, y\right)-G\left(t-s_{1}, x, y\right)\right) \sigma\left(u\left(s_{1}, y\right)\right) d y\right|^{n}\right], \\
& I_{3}(n)=E\left[\left|\int_{s_{1}}^{l} \int_{\mathcal{O}} G(l-\lambda, z, \zeta) B(\lambda, \zeta)\left(X_{s_{1}}(\lambda, \zeta)-X_{s_{2}}(\lambda, \zeta)\right) d \lambda d \zeta\right|^{n}\right], \\
& I_{4}(n)=E\left[\left|\int_{s_{2}}^{s_{1}} \int_{\mathcal{O}} G(l-\lambda, z, \zeta) B(\lambda, \zeta) X_{s_{2}}(\lambda, \zeta) d \lambda d \zeta\right|^{n}\right], \\
& I_{5}(n)=E\left[\left.|| \int_{s_{1}}^{l} \int_{\mathcal{O}} G(l-\lambda, z, \zeta) S(\lambda, \zeta)\left(X_{s_{1}}(\lambda, \zeta)-X_{s_{2}}(\lambda, \zeta)\right) d W(\lambda, \zeta)\right|^{n}\right],
\end{aligned}
$$




$$
I_{6}(n)=E\left[\left.|| \int_{s_{2}}^{s_{1}} \int_{\mathcal{O}} G(l-\lambda, z, \zeta) S(\lambda, \zeta) d W(\lambda, \zeta)\right|^{n}\right] .
$$

SteP 2. Let us estimate $I_{1}(n)$. We have, by Hölder's inequality, for $0<\varepsilon<1$,

$$
\begin{aligned}
I_{1}(n) \leq & \left|\int_{Q} G^{\left(\frac{n}{n-1}\right) \varepsilon}\left(t-s_{2}, x, y\right) d y\right|^{n-1} \\
& \times \int_{Q} G^{(1-\varepsilon) n}\left(t-s_{2}, x, y\right) E\left[\left|\sigma\left(u\left(s_{2}, y\right)\right)-\sigma\left(u\left(s_{1}, y\right)\right)\right|^{n}\right] d y .
\end{aligned}
$$

By Theorem 3.1(iii) and the Lipschitz property of $\sigma$, we know that for $n$ large enough and any $y \in \mathcal{O}$, setting $\widehat{n}=[2(n-1) /(n-2)]-1$, we have

$$
E\left[\left|\sigma\left(u\left(s_{2}, y\right)\right)-\sigma\left(u\left(s_{1}, y\right)\right)\right|^{n}\right] \leq c\left|s_{1}-s_{2}\right|^{\left(1-\frac{d}{\gamma} \widehat{n}\right) \frac{n-2}{2}} .
$$

Hence, choosing $\varepsilon=(n-1) / n$, we get

$$
I_{1}(n) \leq c\left|\int_{Q} G\left(t-s_{2}, x, y\right) d y\right|^{n}\left|s_{1}-s_{2}\right|^{\left(1-\frac{d}{\gamma} \widehat{n}\right) \frac{n-2}{2}} \leq c\left|s_{1}-s_{2}\right|^{\left(1-\frac{d}{\gamma} \widehat{n}\right) \frac{n-2}{2}}
$$

using hypothesis (H3).

STEP 3. Let us compute $I_{2}(n)$. As in Step 1, we have

$$
\begin{aligned}
I_{2}(n) \leq & c\left(\int_{Q}\left|G\left(t-s_{2}, x, y\right)-G\left(t-s_{1}, x, y\right)\right| d y\right)^{n-1} \\
& \times\left(\int_{Q}\left|G\left(t-s_{2}, x, y\right)-G\left(t-s_{1}, x, y\right)\right| E\left[\left|\sigma\left(u\left(s_{1}, y\right)\right)\right|^{n}\right] d y\right),
\end{aligned}
$$

and noting that from Theorem 3.1(ii),

$$
\sup _{t \in[0, T]} \sup _{y \in \mathcal{O}} E\left[|\sigma(u(s, y))|^{n}\right] \leq c,
$$

we get

$$
I_{2}(n) \leq c\left(\int_{Q}\left|G\left(t-s_{2}, x, y\right)-G\left(t-s_{1}, x, y\right)\right| d y\right)^{n} .
$$

Recall that $s_{1}>s_{2}$. Applying (H5) with $s=s_{1}-s_{2}, r=\left(t-s_{1}\right) /\left(s_{1}-s_{2}\right)$, we get

$$
\begin{aligned}
& \left|G\left(t-s_{2}, x, y\right)-G\left(t-s_{1}, x, y\right)\right| \\
& \quad=\left|(2 \pi)^{-d} \int_{\mathbb{R}^{d}} \exp (i(x-y))\left[g\left(t-s_{2}, y, \bar{\xi}\right)-g\left(t-s_{1}, y, \bar{\xi}\right)\right] d \bar{\xi}\right| \\
& \quad \leq c\left(s_{1}-s_{2}\right)^{1-d / \gamma}\left(t-s_{1}\right)^{-1} \leq c\left(s_{1}-s_{2}\right)^{1-d / \gamma} t^{-1},
\end{aligned}
$$


since $\left(t-s_{1}\right)^{-1} \leq 2 / t$. Hence, adding the previous results shows that there exists a $\beta \in(0,1)$ such that for $n$ large enough,

$$
I_{1}(n)+I_{2}(n) \leq c\left(s_{1}-s_{2}\right)^{\beta n} t^{-\delta n}
$$

for any $s_{1}, s_{2} \in[0, t / 2]$.

Step 4. Set, for $\lambda \in[0, t]$ and a fixed $\delta>0$,

$$
\begin{aligned}
& F_{n}(\lambda)=\sup \left\{E\left[\left|X_{s_{2}}(\lambda, \zeta)-X_{s_{1}}(\lambda, \zeta)\right|^{n}\right]:\right. \\
& \left.\quad \zeta \in \mathcal{O}, s_{1}, s_{2} \in[0, t / 2],\left|s_{1}-s_{2}\right| \leq \delta\right\} .
\end{aligned}
$$

We will estimate $I_{3}(n)$ and $I_{5}(n)$ in terms of $F_{n}(\theta)$. Since the stochastic part is less easy to treat, we will concentrate on $I_{5}(n)\left(I_{3}(n)\right.$ can be estimated with similar arguments): by Burkholder's inequality, supposing $s_{2}<s_{1}<l$, we have

$$
\begin{aligned}
I_{5}(n) & \leq E\left[\left|\int_{s_{1}}^{l} \int_{\mathcal{O}} G^{2}(l-\lambda, z, \zeta) S^{2}(\lambda, \zeta)\left(X_{s_{1}}(\lambda, \zeta)-X_{s_{2}}(\lambda, \zeta)\right)^{2} d \lambda d \zeta\right|^{n / 2}\right] \\
& \leq c E\left[\left|\int_{s_{1}}^{l} \int_{\mathcal{O}} G^{2}(l-\lambda, z, \zeta)\left(X_{s_{1}}(\lambda, \zeta)-X_{s_{2}}(\lambda, \zeta)\right)^{2} d \lambda d \zeta\right|^{n / 2}\right],
\end{aligned}
$$

since $S$ is a bounded process. Applying Hölder's inequality with $q=2 / n$ and $q^{\prime}=n /(n-2)$ leads to

$$
\begin{aligned}
I_{5}(n) \leq & c\left(\int_{s_{1}}^{l} \int_{\mathcal{O}} G^{n \varepsilon}(l-\lambda, z, \zeta) E\left[\left|X_{s_{1}}(\lambda, \zeta)-X_{s_{2}}(\lambda, \zeta)\right|^{n}\right] d \lambda d \zeta\right) \\
& \times\left(\int_{s_{1}}^{t} \int_{\mathcal{O}} G^{\frac{2 n}{n-2}(1-\varepsilon)}(l-\lambda, z, \zeta) d \lambda d \zeta\right)^{(n-2) / 2},
\end{aligned}
$$

and choosing once again $\varepsilon=2 / n$, we have

$$
\left|I_{5}(n)\right| \leq c \int_{0}^{l}(l-\lambda)^{-d / \gamma} F_{n}(\lambda) d \lambda .
$$

Similarly, we find

$$
\left|I_{3}(n)\right| \leq c \int_{0}^{l} F_{n}(\lambda) d \lambda .
$$

STEP 5. Using again Burkholder's and Hölder's inequalities, we get

$$
\begin{aligned}
\left|I_{6}(n)\right| \leq & c\left(\int_{s_{2}}^{s_{1}} \int_{\mathcal{O}} G^{2}(l-\lambda, z, \zeta) E\left[\left|X_{s_{2}}(\lambda, \zeta)\right|^{2}\right] d \lambda d \zeta\right) \\
& \times\left(\int_{s_{2}}^{s_{1}} \int_{\mathcal{O}} G^{2}(l-\lambda, z, \zeta) d \lambda d \zeta\right)^{(n-2) / 2}
\end{aligned}
$$


and by inequality (10),

$$
\begin{aligned}
\left|I_{6}(n)\right| & \leq c\left(\int_{s_{2}}^{s_{1}} \int_{\mathcal{O}} G^{2}(l-\lambda, z, \zeta) d \lambda d \zeta\right)^{n / 2} \leq c\left(\int_{s_{2}}^{s_{1}}(l-\lambda)^{-d / \gamma} d \lambda\right)^{n / 2} \\
& \leq c\left(s_{1}-s_{2}\right)^{(1-d / \gamma) n / 2} .
\end{aligned}
$$

Similarly,

$$
\left|I_{4}(n)\right| \leq c\left|s_{1}-s_{2}\right|^{n / 2},
$$

and adding the previous results, we get

$$
F_{n}(l) \leq\left(\delta^{-\beta n} t^{-n}+\int_{0}^{l}(l-\lambda)^{-d / \gamma} F_{n}(\lambda) d \lambda\right) .
$$

We can apply a result of D. Henry [5, Lemma 7.1.1] to get, for a constant $c>0$,

$$
\sup _{l \in[0, t]} F_{n}(l) \leq c \delta^{-\beta n} t^{-n} .
$$

In particular, for $l=t$, we get

$$
\sup \left\{E\left[\left|r_{s_{1}}(t, x)-r_{s_{2}}(t, x)\right|^{n}\right]: s_{1}, s_{2} \in[0, t / 2],\left|s_{1}-s_{2}\right| \leq \delta\right\} \leq c \delta^{-\beta n} t^{-n},
$$

and, for $n$ such that $\beta n>1$, Kolmogorov's continuity criterion gives us the continuity in $s$ of the process $\left\{r_{s}(t, x): s \in[0, t / 2]\right\}$.

4.2. Existence of a density. We now show the main result of this paper, whose proof follows closely the lines of [14] and [13, Theorem 2.4.4].

Theorem 4.3. Assume hypotheses (H1) to (H7), and suppose $\sigma\left(u_{0}(y)\right)$ $\neq 0$ for some $y_{0} \in \mathcal{O}$. Then the law of $u(t, x)$ is absolutely continuous with respect to the Lebesgue measure for any $(t, x) \in(0, T] \times \mathcal{O}$.

Pro of. We divide the proof in several oesteps.

STEP 1. Suppose that $\sigma\left(u_{0}\left(y_{0}\right)\right)>0$ for some $y_{0} \in \mathcal{O}\left(\right.$ the case $\sigma\left(u_{0}\left(y_{0}\right)\right)$ $<0$ can be treated similarly). Fix $(t, x) \in(0, T] \times \mathcal{O}$. According to Theorem 2.3 , we have to prove that

$$
\int_{0}^{t} \int_{\mathcal{O}}\left|D_{s, y} u(t, x)\right|^{2} d y d s>0 \quad \text { a.s. }
$$

By continuity of $u_{0}$, there is a compact set $Q \subset \mathcal{O}$ such that $\sigma\left(u_{0}(y)\right) \geq$ $2 \delta>0$ for every $y \in Q$, and by the continuity of $\sigma$ and $u$, there exists a stopping time $\tau_{1}>0$ such that $\sigma(u(s, y)) \geq \delta>0$ for every $y \in Q, s \leq \tau_{1}$. Set then

$$
r_{s}(t, x) \equiv \int_{Q} D_{s, y} u(t, x) d y
$$


In order to show (11), it is sufficient to prove that there exists a stopping time $\tau_{2}$ such that $\tau_{2}>0$ a.s. and $r_{s}(t, x)>0$ for any $0 \leq s \leq \tau_{2}$. Moreover by Lemma 4.2 , the process $\left\{r_{s}(t, x): s \in[0, t / 2]\right\}$ is almost surely continuous. Hence, it is sufficient to show that $r_{0}(t, x)>0$. Set $v(t, x)=r_{0}(t, x)$. Then $v$ satisfies the equation

$$
\begin{aligned}
v(t, x)= & \int_{Q} G(t, x, y) \sigma\left(u_{0}(y)\right) d y \\
& +\int_{0}^{t} \int_{\mathcal{O}} G(t-\theta, x, \xi) B(\theta, \xi) v(\theta, \xi) d \theta d \xi \\
& +\int_{0}^{t} \int_{\mathcal{O}} G(t-\theta, x, \xi) S(\theta, \xi) v(\theta, \xi) d W(\theta, \xi) .
\end{aligned}
$$

We will show that $v(t, x)>0$ almost surely.

Step 2. For some $c>0$ to be specified later, set $\chi(t, x)=e^{c t} v(t, x)$. Then $v(t, x)>0$ if and only if $\chi(t, x)>0$. Note that $\chi$ is a solution of the equation

$$
\left\{\begin{aligned}
d \chi(t, x)= & {[L \chi(t, x)+(B(t, x)+c) \chi(t, x)] d t } \\
& +S(t, x) \chi(t, x) d W(t, x) \\
\chi(0, x)= & \sigma\left(u_{0}(x)\right) \mathbf{1}_{Q}(x)
\end{aligned}\right.
$$

If we take $c$ large enough, then, by boundedness of $B$, we see that $B+c$ is a positive process. Moreover, $\sigma\left(u_{0}(x)\right) \geq \delta$ on $Q$, and by linearity of equation (13), we can suppose $\delta=1$. Hence, by the comparison theorem 3.3 for solutions of a pseudodifferential equation, we only have to show that $v(t, x)>0$ when $v$ is the solution to the integral equation

$$
v(t, x)=\int_{Q} G(t, x, \xi) d \xi+\int_{0}^{t} \int_{\mathcal{O}} G(t-\theta, x, \xi) S(\theta, \xi) v(\theta, \xi) d W(\theta, \xi) .
$$

STEP 3. We know that $x \in Q^{d}$ for some $d>0$, where for any $l>0$,

$$
Q^{l}=\{z \in \mathcal{O}: d(z, Q)<l\} .
$$

Set also

$$
\alpha=\inf \left\{\frac{1}{2} \int_{Q} G(s, y, z) d z: y \in Q^{d}, s \in[0, T]\right\} \wedge 1 .
$$

By hypothesis (H6), we know that $\alpha>0$. For $k=1, \ldots, m$, set $t_{k}^{m}=k / m$ and

$$
E_{k}=\left\{v\left(t_{k}^{m}, y\right) \geq \alpha^{k} \mathbf{1}_{Q^{k m / d}}(y), \forall y \in \mathcal{O}\right\} .
$$

Suppose that for any $\delta>0$, there exists an $m_{0}>1$ such that if $m \geq m_{0}$, 
then for any $0 \leq k \leq m-1$,

$$
P\left(E_{k+1}^{c} \mid E_{1} \cap \ldots \cap E_{k}\right) \leq \delta / m .
$$

As a direct consequence of (14), we get

$$
\begin{aligned}
P(v(t, x)>0) \geq & P\left(v(t, y) \geq \alpha^{m} \mathbf{1}_{Q^{d}}(y), \forall y \in \mathcal{O}\right) \geq P\left(\bigcup_{k=1}^{m} E_{k}\right) \\
= & P\left(E_{m} \mid E_{m-1} \cap \ldots \cap E_{1}\right) \\
& \times P\left(E_{m-1} \mid E_{m-2} \cap \ldots \cap E_{1}\right) \ldots P\left(E_{1}\right) \\
\geq & (1-\delta / m)^{m} \geq 1-\delta,
\end{aligned}
$$

and as $\delta$ is arbitrary, we have $P(v(t, x)>0)=1$.

STEP 4. Let us now check inequality (14). For $s \in\left[t_{k}^{m}, t_{k+1}^{m}\right)$ and $y \in \mathcal{O}$, we have

$$
\begin{aligned}
v(s, y)= & \int_{\mathcal{O}} G\left(s-t_{k}^{m}, y, \eta\right) v\left(t_{k}^{m}, \eta\right) d \eta \\
& +\int_{t_{k}^{m}}^{s} \int_{\mathcal{O}} G(s-\theta, y, \eta) S(\theta, \eta) v(\theta, \eta) d W(\theta, \eta) .
\end{aligned}
$$

On the set $E_{1} \cap \ldots \cap E_{k}$, by the comparison theorem, we have $v(s, y) \geq x(s, y)$ where $x=\left\{x(s, y): s \in\left[t_{k}^{m}, t_{k+1}^{m}\right], y \in \mathcal{O}\right\}$ is a process defined by

$$
\begin{aligned}
x(s, y)= & \alpha^{k} \int_{Q^{k d / m}} G\left(s-t_{k}^{m}, y, \eta\right) d \eta \\
& +\int_{t_{k}^{m}}^{s} \int_{\mathcal{O}} G(s-\theta, y, \eta) S(\theta, \eta) x(\theta, \eta) d W(\theta, \eta) .
\end{aligned}
$$

Hence,

$$
P\left(E_{k+1} \mid E_{1} \cap \ldots \cap E_{k}\right) \geq P\left(x\left(t_{k+1}^{m}, y\right) \geq \alpha^{k+1}, \forall y \in Q^{(k+1) d / m}\right),
$$

where we have omitted the conditioning on $E_{1}, \ldots, E_{k}$ on the right hand side (it only affects the values of $S(\theta, \eta)$ ). For any $y \in Q^{(k+1) d / m}$, we have

$$
\int_{Q^{k d / m}} G\left(t_{k}^{m}, y, \eta\right) d \eta \geq 2 \alpha
$$

Set, for $y \in \mathcal{O}$,

$$
\phi_{k+1}(y)=\int_{t_{k}^{m}}^{t_{k+1}^{m}} \int_{\mathcal{O}} G\left(t_{k+1}^{m}-\theta, y, \eta\right) S(\theta, \eta) \frac{x(\theta, \eta)}{\alpha^{k}} d W(\theta, \eta) .
$$

Using inequality (16) and the definition of $x$ given by (15), we obtain, for 
any $n>1$,

$$
\begin{aligned}
P\left(E_{k+1}^{\mathrm{c}} \mid E_{1} \cap \ldots \cap E_{k}\right) & \leq P\left(\sup _{y \in Q^{(k+1) d / m}}\left|\phi_{k+1}(y)\right|>\alpha\right) \\
& \leq \alpha^{-n} E\left[\sup _{y \in Q^{d}}\left|\phi_{k+1}(y)\right|^{n}\right] .
\end{aligned}
$$

STEP 5. Let us give an estimate for the moments of $\phi_{k+1}$, using Garsia's Lemma: for $y_{1}, y_{2} \in Q^{d}$, using once more Burkholder's and Hölder's inequalities, we get

$$
\begin{aligned}
& E\left[\left|\phi_{k+1}\left(y_{1}\right)-\phi_{k+1}\left(y_{2}\right)\right|^{n}\right] \\
& \leq c \int_{t_{k}^{m}}^{t_{k+1}^{m}} \int_{\mathcal{O}}\left(G\left(t_{k+1}^{m}-\theta, y_{1}, \eta\right)-G\left(t_{k+1}^{m}-\theta, y_{2}, \eta\right)\right)^{2} E\left[\left|\alpha^{-k} x(\theta, \eta)\right|^{n}\right] d \theta d \eta \\
& \quad \times\left(\int_{t_{k}^{m}}^{t_{k+1}^{m}} \int_{\mathcal{O}}\left(G\left(t_{k+1}^{m}-\theta, y_{1}, \eta\right)-G\left(t_{k+1}^{m}-\theta, y_{2}, \eta\right)\right)^{2} d \theta d \eta\right)^{(n-2) / 2} .
\end{aligned}
$$

Note that, by linearity of equation (15), we have, for any $n>1$,

$$
\sup _{k \geq 1} \sup _{\eta \in \mathcal{O}} \sup _{\theta \in\left[t_{k}^{m}, t_{k+1}^{m}\right]} E\left[\left|\alpha^{-k} x(\theta, \eta)\right|^{n}\right] \leq c<\infty,
$$

where the constant $c$ does not depend on $m$. Hence, for a constant $c>0$,

$$
\begin{aligned}
& E\left[\left|\phi_{k+1}\left(y_{1}\right)-\phi_{k+1}\left(y_{2}\right)\right|^{n}\right] \\
& \quad \leq c\left(\int_{t_{k}^{m}}^{t_{k+1}^{m}} \int_{\mathcal{O}}\left(G\left(t_{k+1}^{m}-\theta, y_{1}, \eta\right)-G\left(t_{k+1}^{m}-\theta, y_{2}, \eta\right)\right)^{2} d \theta d \eta\right)^{n / 2} \\
& \quad \leq c\left(\int_{0}^{t / m} \int_{\mathcal{O}}\left(G\left(\theta, y_{1}, \eta\right)-G\left(\theta, y_{2}, \eta\right)\right)^{2} d \theta d \eta\right)^{n / 2} .
\end{aligned}
$$

STEP 6. Let us get an estimate for the right hand side of (17). Take $\alpha=(\gamma-d) / \gamma$, and note that $\alpha<(\gamma-d) \wedge 1$. Then, using hypothesis (H4) and the fact that for $0<\beta<1$ and $k, x \in \mathbb{R}^{d}$,

$$
\left|e^{i k x}-1\right|<c_{\beta}|k|^{\beta}|x|^{\beta},
$$

we get

$$
\begin{aligned}
\mid G\left(\theta, y_{1}, \eta\right) & -G\left(\theta, y_{2}, \eta\right) \mid \\
= & \left|(2 \pi)^{-d} \int_{\mathbb{R}^{d}}\left[\exp \left(i\left(y_{1}-\eta\right) \bar{\eta}\right)-\exp \left(i\left(y_{2}-\eta\right) \bar{\eta}\right)\right] g(\theta, \eta, \bar{\eta}) d \bar{\eta}\right| \\
\leq & c \int_{\mathbb{R}^{d}}\left|y_{1}-y_{2}\right|^{\alpha}|\bar{\eta}|^{\alpha}|g(\theta, \eta, \bar{\eta})| d \bar{\eta}
\end{aligned}
$$




$$
\begin{aligned}
& =c\left|y_{1}-y_{2}\right|^{\alpha} \int_{\mathbb{R}^{d}}|\bar{\eta}|^{\alpha}|g(\theta, \eta, \bar{\eta})| d \bar{\eta} \\
& =c\left|y_{1}-y_{2}\right|^{\alpha} \theta^{-(d+\alpha) / \gamma} \int_{\mathbb{R}^{d}}|x|^{\alpha}\left|g\left(\theta, \eta, \theta^{-1 / \gamma} x\right)\right| d x \\
& \leq c\left|y_{1}-y_{2}\right|^{\alpha} \theta^{-(d+\alpha) / \gamma} .
\end{aligned}
$$

Note that $(d+\alpha) / \gamma<1$. Hence, by hypotheses (H3),

$$
\begin{aligned}
& \int_{0}^{t / m} \int_{\mathcal{O}}\left(G\left(\theta, y_{1}, \eta\right)-G\left(\theta, y_{2}, \eta\right)\right)^{2} d \theta d \eta \\
& \quad \leq c\left|y_{1}-y_{2}\right|^{\alpha} \int_{0}^{t / m} \theta^{-(d+\alpha) / \gamma}\left(\int_{\mathcal{O}}\left|G\left(\theta, y_{1}, \eta\right)-G\left(\theta, y_{2}, \eta\right)\right| d \eta\right) d \theta \\
& \quad \leq c\left|y_{1}-y_{2}\right|^{\alpha}(t / m)^{\beta}
\end{aligned}
$$

with

$$
0<\beta \equiv(\gamma-d)(\gamma-1) / \gamma^{2}<1
$$

In particular, if $y_{1}, y_{2} \in Q^{d}$, then

$$
E\left[\left|\phi_{k+1}\left(y_{1}\right)-\phi_{k+1}\left(y_{2}\right)\right|^{n}\right] \leq c\left|y_{1}-y_{2}\right|^{\alpha n / 2}(t / m)^{\beta n / 2} .
$$

Since $n$ can be taken artibrarily large, we can choose $\alpha n / 2>d$ and $\beta n / 2$ $>1$. We can also choose a $Q^{d}$ satisfying the cone condition. Hence, by Theorem 3.4, we find that almost surely, for some $0<l<\alpha n / 2-d$ and any $y_{1}, y_{2} \in Q^{d}$,

$$
\left|\phi_{k+1}\left(y_{1}\right)-\phi_{k+1}\left(y_{2}\right)\right|^{n} \leq c_{1}\left|y_{1}-y_{2}\right|^{l}(t / m)^{\beta n / 2} \Gamma
$$

where $c_{1}$ is a positive constant and $\Gamma$ is a random variable such that

$$
E[\Gamma] \leq c_{2},
$$

with $0<c_{2}<\infty$. Since the set $Q^{d}$ is bounded, and since $\phi_{k}(y)$ has moments of any order for every $y \in Q^{d}$, the following holds for sufficiently large $n$ :

$$
E\left[\sup _{y \in Q^{d}}\left|\phi_{k+1}(y)\right|^{n}\right] \leq c / m \text {. }
$$

Thus

$$
P\left(E_{k+1}^{\mathrm{c}} \mid E_{1} \cap \ldots \cap E_{k}\right) \leq c / m
$$

which ends the proof.

\section{References}

[1] V. Bally and E. Pardoux, Malliavin calculus for white noise driven parabolic SPDEs, Potential Anal. 9 (1998), 27-64. 
[2] Z. Brzeźniak, On stochastic convolution in Banach spaces and applications, Stochastics Stochastics Rep. 61 (1997), 245-295.

[3] G. Da Prato and J. Zabczyk, Stochastic Equations in Infinite Dimensions, Cambridge Univ. Press, 1992.

[4] C. Donati et E. Pardoux, EDPS réfléchies et calcul de Malliavin, Bull. Sci. Math. 121 (1997), 405-422.

[5] D. Henry, Geometric Theory of Semilinear Parabolic Equations, Lecture Notes in Math. 840, Springer, Berlin, 1981.

[6] N. Jacob, Feller semigroups, Dirichlet forms, and pseudo-differential operators, Forum Math. 4 (1992), 433-446.

[7] G. Kallianpur and J. Xiong, Large deviation for a class of stochastic differential equations, Ann. Probab. 24 (1996), 320-345.

[8] P. Kotelenez, Existence, uniqueness and smoothness for a class of function valued stochastic partial differential equations driven by space-time white noise, Stochastics Stochastics Rep. 41 (1992), 177-199.

[9] —, A class of function and density valued stochastic partial differential equations driven by space-time white noise, to appear.

[10] N. Lanjri and D. Nualart, Burgers equation driven by space-time white noise: absolute continuity of the solution, Stochastics Stochastics Rep. 66 (1999), 273-292.

[11] D. Márquez and M. Sanz, Taylor expansion of the density of the law in a stochastic heat equation, Collect. Math. 49 (1998), 399-415.

[12] A. Millet and M. Sanz, A stochastic wave equation in two space dimension: smoothness of the law, Ann. Probab. 27 (1999), 803-844.

[13] D. Nualart, The Malliavin Calculus and Related Topics, Springer, Berlin, 1995.

[14] E. Pardoux and T. Zhang, Absolute continuity of the law of the solution of a parabolic SPDE, J. Funct. Anal. 112 (1993), 447-458.

[15] S. Peszat and J. Zabczyk, Stochastic evolution equations with a spatially homogeneous Wiener process, Stochastic Process. Appl. 72 (1997), 187-204.

[16] J. Seidler, Da Prato-Zabczyk's maximal inequality revisited I, Math. Bohem. 118 (1993), 67-106.

[17] F. Treves, Introduction to Pseudodifferential and Fourier Integral Operators, Plenum, New York, 1982.

[18] J. Walsh, An introduction to stochastic partial differential equations, in: Ecole d'été de Probabilité de Saint-Flour XIV, Lecture Notes in Math. 1180, Springer, Berlin, 1986, 265-439.

Samy Tindel

Département de Mathématiques-Institut Galilée

Université Paris 13

Avenue J. B. Clément

93430 Villetaneuse, France

E-mail: tindel@math.univ-paris13.fr

Received on 9.3.1999;

revised version on 10.11.1999 